\title{
BMJ
}

\section{Use of intensive case management to reduce time in hospital in people with severe mental illness: systematic review and meta-regression}

\author{
Tom Burns, professor of social psychiatry, ${ }_{1}^{1}$ Jocelyn Catty, research fellow, ${ }^{2}$ Michael Dash, research \\ assistant, ${ }^{2}$ Chris Roberts, statistician, ${ }^{3}$ Austin Lockwood, research nurse, ${ }^{4}$ Max Marshall, professor of \\ community psychiatry ${ }^{4}$
}

'University of Oxford, Warneford Hospital, Oxford OX3 7JX

${ }^{2}$ Division of Mental Health, St George's, University of London, London SW17 ORE

${ }^{3}$ School of Epidemiology and Health Sciences, University of Manchester, Manchester

${ }^{4}$ Division of Psychiatry and Behavioural Sciences, University of Manchester, Preston

Correspondence to: T Burns Tom.burns@psych.ox.ac.uk

doi:10.1136/bmj.39251.599259.55

\section{ABSTRACT}

Objectives To explain why clinical trials of intensive case management for people with severe mental illness show such inconsistent effects on the use of hospital care.

Design Systematic review with meta-regression techniques applied to data from randomised controlled trials.

Data Sources Cochrane central register of controlled trials, CINAHL, Embase, Medline, and PsychINFO databases from inception to January 2007. Additional anonymised data on patients were obtained for multicentre trials.

Review methods Included trials examined intensive case management compared with standard care or low intensity case management for people with severe mental illness living in the community. We used a fidelity scale to rate adherence to the model of assertive community treatment. Multicentre trials were disaggregated into individual centres with fidelity data specific for each centre. A multivariate meta-regression used mean days per month in hospital as the dependent variable.

Results We identified 1335 abstracts with a total of 5961 participants. Of these, 49 were eligible and 29 provided appropriate data. Trials with high hospital use at baseline (before the trial) or in the control group were more likely to find that intensive case management reduced the use of hospital care (coefficient $-0.23,95 \%$ confidence interval -0.36 to -0.09 , for hospital use at baseline; $-0.44,-0.57$ to -0.31 , for hospital use in control groups). Case management teams organised according to the model of assertive community treatment were more likely to reduce the use of hospital care (coefficient $-0.31,-0.59$ to -0.03), but this finding was less robust in sensitivity analyses and was not found for staffing levels recommended for assertive community treatment. Conclusions Intensive case management works best when participants tend to use a lot of hospital care and less well when they do not. When hospital use is high, intensive case management can reduce it, but it is less successful when hospital use is already low. The benefits of intensive case management might be marginal in settings that have already achieved low rates of bed use, and team organisation is more important than the details of staffing. It might not be necessary to apply the full model of assertive community treatment to achieve reductions in inpatient care.

\section{INTRODUCTION}

Modern mental health services try to ensure that people with severe mental illness spend the minimum amount of time in hospital because unnecessary hospital care is wasteful, stigmatising, and disliked by patients. To achieve this goal, mental health services increasingly use intensive case management to care for severely mentally ill people at high risk of readmission. Such programmes have been set up across the United States healthcare system ${ }^{1}$ and widely adopted in Canada, Australia, and Europe. ${ }^{23}$ In the United Kingdom, the national service framework has authorised the setting up of 170 high fidelity assertive outreach teams. ${ }^{4}$

Assertive outreach was previously referred to in the UK as intensive case management, a somewhat broader term emphasising small caseloads. It is the term used for the UK application of the North American service assertive community treatment, a carefully specified approach to case management, ${ }^{2}$ which includes daily team meetings, case sharing, 24 hour availability, and multidisciplinary working with doctors as full team members.

Under intensive case management, each person with severe mental illness at high risk of readmission is allocated a nurse, social worker, or other clinician (a "case manager") who carries a small caseload of between 10 and 20 patients. This case manager takes primary responsibility for keeping contact with the patient, assessing their needs, and ensuring that these needs are met. ${ }^{5}$ Intensive case management is one of the most thoroughly evaluated non-pharmaceutical interventions in psychiatry, but numerous trials over the past 35 years have failed to show that it consistently reduces the use of hospital care. While some trials have shown a large reduction, others have found no effect, and some have shown a significant increase.

This inconsistent effect on hospital care might be explained by differences in the contexts in which the 


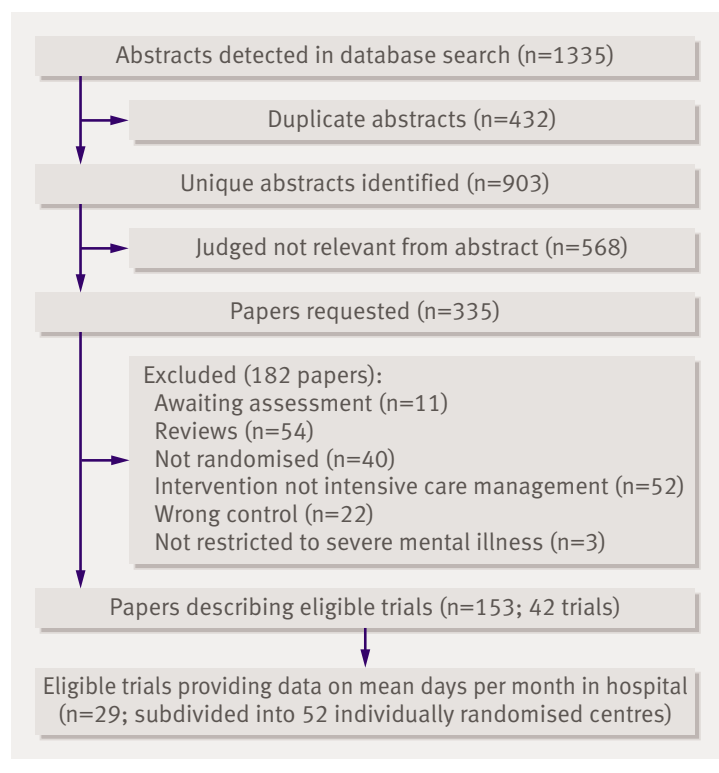

Fig 1| Process of identification of studies included in review

trials were conducted. Some have suggested that intensive case management is effective only in the US, with the presumption that care in US control groups is inferior to that in state funded welfare systems such as in the UK. ${ }^{3}$ Alternatively, case managers in these studies might not be working as a team, as advocated by the model of assertive community treatment. ${ }^{6}$ Others have proposed that intensive case management is less effective in settings where control groups are contaminated with case management practices (a particular feature of more recent trials). ${ }^{7-9}$ Another intriguing suggestion is that intensive case management is effective only against a background of high reliance on hospital care. $^{1011}$

An alternative hypothesis is that the inconsistent results are caused by different models of intensive case management being practised in different trials. In particular it has been suggested that hospital care was reduced only in trials where assertive community treatment was practised.

We aimed to test all these hypotheses by applying meta-regression techniques to data from randomised controlled trials of intensive case management.

\section{METHODS}

Data sources

We identified randomised controlled trials available for review by January 2007 that had compared intensive case management (caseload up to and including 20) with standard care (from a community mental health team or outpatient clinic) or low intensity case management (caseload greater than 20) in people with severe mental disorder living in the community. We defined severe mental disorder as schizophrenia or schizophrenia-like disorder, bipolar disorder, or depression with psychotic features. The search strategy (see www.psychiatry.ox.ac.uk/socpsych/bmjmrtable) was updated from that of two previous reviews. ${ }^{1213}$ We searched CINAHL (January 1982-January 2007), the Cochrane central register of controlled trials (issue 4, 2006), Embase (January 1980-January 2007), Medline (January 1966-January 2007), and PsycINFO (January 1872-January 2007) and examined the reference lists of all relevant studies and reviews.

\section{Study selection}

We excluded trials in which the experimental intervention was an acute crisis team or if the control condition was hospital admission, remaining in hospital, or an alternative form of intensive case management. We also excluded those in which most participants were aged under 18 or over 65 or had a primary diagnosis of organic brain disorder or learning disability. Two authors (MM and AL or JC and MD) screened each abstract and sought potentially relevant articles. They independently reviewed articles and constructed a table of included studies. Any disagreements were resolved by discussion with a third reviewer (JC or $\mathrm{TB}$, respectively). We categorised each included trial for allocation concealment according to the Cochrane Collaboration Handbook ${ }^{14}$ and included only trials rated $\mathrm{A}$ or $\mathrm{B}$.

\section{Data extraction}

The dependent variable in the meta-regression was time in hospital, defined as mean number of days per month in hospital. Data on the dependent variable were eligible only if provided on an intention to treat basis and available for more than half the trial participants. Two independent reviewers (MM and $\mathrm{AL}$ ) extracted data from trial reports and cross checked data by double entry. If possible, we calculated the

Relation between hypotheses tested and covariates used in the meta-regression (shown in parentheses)

Variation in the outcome of hospital use between trials of intensive case management is explained by:

Lack of team working as recommended in assertive community treatment in the case management team in the trial (team structure and organisation subscale of IFACT)

Lack of personnel resources as recommended in assertive community treatment in the case management team in the trial (team membership subscales of IFACT)

Factors related to trial context such as:

Control treatments improving over time (year the study started)

Control treatment from US healthcare system (country in which study took place)

High rates of hospital use in the setting where the trial was conducted (mean days per month in hospital for all participants in the two years before trial)

Case management practices present in control group (degree of low intensity case management in control group)

Publication bias (size of trial) 
dependent variable on the basis of a 24 month followup period as this offered the best trade off between length of follow-up and attrition of participants. When a trial did not report 24 month data, we calculated the dependent variable from the nearest available follow-up point. When a trial reported a mean but no $\mathrm{SD}$, we imputed the missing parameter using a regression analysis of SD against mean, based on data from trials that provided SDs.

We also noted the degree of low intensity case management in the control group (all, some, or none), the country where trial took place (US or non-US); mean days per month in hospital for participants in the two years before the study began (baseline hospital use); the year the study began; trial size; and a rating of fidelity of the intervention to assertive community treatment on the "team membership" and "team structure and organisation" subscales of the index of fidelity to assertive community treatment (IFACT). ${ }^{15}$ This index was derived from a survey of 20 clinical experts in assertive community treatment and validated in a survey of 18 programmes. The team membership subscale comprises four items: ratio of patients to staff, total size of the team, and the extent of psychiatric and nursing input to the team. The structure and organisation subscale comprises seven items: whether the team is the primary source of care for its patients, is situated away from the hospital, meets daily, shares responsibility for caseloads, is available 24 hours a day, has a team leader who is also a case manager, and offers unlimited time for its services. We chose the index because the subscales are brief and can be completed from published data. For each item on the index, a score of 1 indicates high fidelity to the model. Fidelity data were obtained from published and unpublished trial reports; direct contact with trialists (who either completed the scale from memory or supplied fidelity data collected contemporaneously); and data previously obtained directly from trialists by a previous review. ${ }^{1617}$ Two raters (MM and AL) independently combined these data into a single fidelity score. Discrepancies were resolved by discussion and, if necessary, by contacting trialists. Items for

Table 1 | Summary of meta-regression analysis of days in hospital per month (52 centres) based on estimates of treatment effect

\begin{tabular}{|c|c|c|c|c|}
\hline & $\begin{array}{c}\text { Coefficient* (days/ } \\
\text { month) }\end{array}$ & SE & $95 \% \mathrm{Cl}$ for coefficient & $P$ value \\
\hline \multicolumn{5}{|l|}{ Fidelity score } \\
\hline Staffing & 0.03 & 0.16 & -0.29 to 0.34 & 0.862 \\
\hline Organisation & -0.444 & 0.14 & -0.72 to -0.17 & 0.002 \\
\hline \multicolumn{5}{|l|}{ Case management } \\
\hline $11-89 \%$ in control & -0.05 & 0.53 & -1.09 to 0.99 & 0.929 \\
\hline$\geq 90 \%$ low intensity & 0.44 & 0.54 & -0.62 to 1.51 & 0.414 \\
\hline Trial size & 0.00 & 0.001 & -0.002 to 0.001 & 0.615 \\
\hline Year (-1990) & 0.07 & 0.06 & -0.05 to 0.19 & 0.257 \\
\hline Country (1=US, $0=$ otherwise) & -0.03 & 0.62 & -1.24 to 1.19 & 0.965 \\
\hline Constant & 1.32 & 0.61 & 0.13 to 2.51 & 0.030 \\
\hline
\end{tabular}

*No variation between centres. Variation between trials was 0.47 .

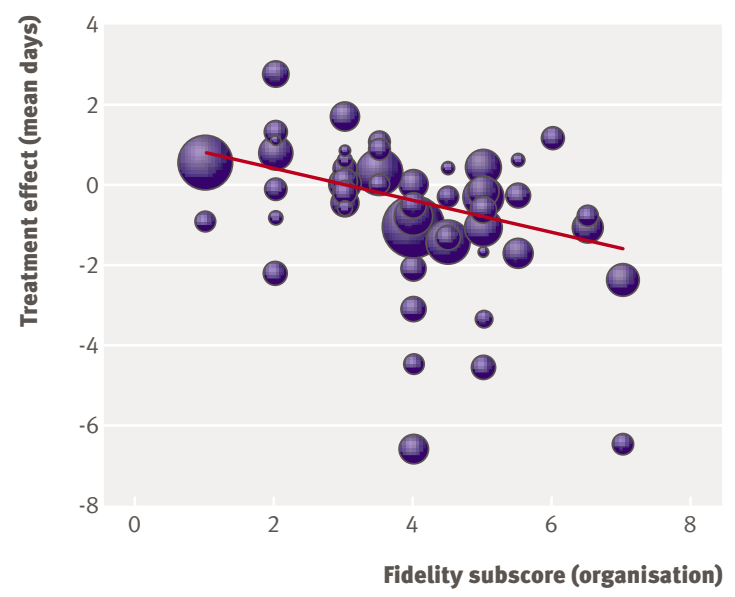

$\overline{\text { Fig } 2 \text { | Scatter plot of IFACT organisation subscore } \boldsymbol{v} \text { mean days }}$ per month in hospital. Each circle is proportional to size of centre it represents. Negative treatment effect indicates that intensive case management achieved reduction in mean days in hospital relative to control

which no data were available were assigned a null value because we assumed that trialists would report their efforts to achieve fidelity to the assertive community treatment model.

Multicentre trials of intensive case management often struggle to implement a uniform approach, ${ }^{18}$ with centres operating at different degrees of fidelity to assertive community treatment. We exploited this to increase the power of the meta-regression by disaggregating multicentre studies into component centres with outcome and fidelity data for each. Trialists provided data either in summary form or as anonymous data on individual patients with the permission of their institutional review boards. We verified independently calculated centre data against original trial reports.

\section{Data synthesis}

The box shows the relation between the covariates and the hypotheses tested. We also included trial size as a covariate to check for publication bias. We used Stata to carry out a random effects meta-analysis with random effects for variation between centres and between studies using a three level random effects model with a numerical constraint on the level 1 variation. This was fitted with generalised linear latent and mixed models (GLLAMM), a Stata algorithm with Gaussian quadrature based maximum likelihood estimation. ${ }^{19}$

Initially, we excluded baseline hospital use as a covariate as this was not available for all centres. Subsequently, we repeated the meta-analysis including only centres for which we had baseline data on hospital use. To reduce the possibility of type 2 errors, this second analysis included only covariates that were found to have a significant association with mean number of days in hospital in the first analysis.

We then carried out two sensitivity analyses on the full dataset to verify our findings. Firstly, we included the mean of the control group as a covariate instead of 


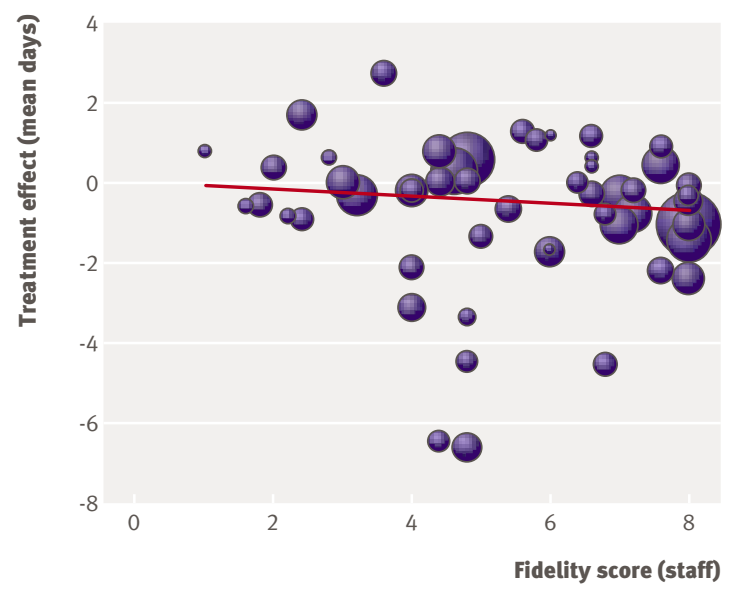

Fig 3 Scatter plot of IFACT team membership subscore $v$ mean days per month in hospital. Each circle is proportional to size of centre it represents. Negative treatment effect indicates that intensive case management achieved reduction in mean days in hospital relative to control

the baseline measure of hospital use. This had the advantage of being available for all centres but was potentially flawed because of mathematical coupling and regression to the mean. Therefore we carried out a second sensitivity analysis by modelling the means of the treatment group rather than the intervention effect. In this model, the relation between the intervention effect and the underlying effect can be assessed by including a random intercept, which assesses the underlying mean for that study or centre, and a random coefficient for intervention whose variance is equivalent to the variance between studies in a conventional meta-analysis of intervention effects. For the covariance between these random effects we can estimate a regression coefficient for the relation between intervention effect and the control group mean.

Finally, we performed two further sensitivity analyses: the first excluded trials with imputed SDs; and the second excluded trials where the Cochrane randomisation category was $\mathrm{B}$.

\section{RESULTS}

Figure 1 summarises the selection of studies for inclusion. The initial search detected 1335 abstracts from which 42 eligible trials, involving 5961 participants, were identified. Twenty nine of these trials provided data on mean days per month in hospital (see www.psychiatry.ox.ac.uk/socpsych/bmjmrtable). ${ }^{\text {w1-w29 }}$ The mean age of participants was 37.9 years, $37 \%$ were female, $66 \%$ had schizophrenia or schizophrenia-like disorder, and 37\% were from ethnic minorities. The mean attrition rate was $4 \%$. Only three included trials had a caseload of more than 15 patients per case manager and 21 had a caseload of 1:10 or less.

\section{Covariates and data on individual patients}

Of the 29 trials, eight were multicentre. We obtained data on individual patients for four of these, involving
1996 participants. ${ }^{\text {w9 } 920 \text { w25 w29 }}$ They subdivided into 23 centres, each with complete fidelity data, but we subsequently excluded one centre (with 87 participants) because fidelity data showed the intervention was low intensity case management. The other four trials ${ }^{\mathrm{w} 4 \mathrm{w} 6 \mathrm{w} 12 \mathrm{w} 15}$ were divided into nine centres by using published data, giving a total of 52 centres for analysis.

We had complete data for low intensity case management in the control group (all, some, or none), country (US or non-US), the year the trial started, and the size of the trial. In 26 centres the control group was standard care without any low intensity case management; in 13 at least $10 \%$ of the control group members received low intensity case management; and in 13 all control group members received low intensity case management. Thirty five centres were from the US (3271 patients); 15 were from the European Union (2494 patients), including 13 from the UK $(n=1541$ patients); and two ( $\mathrm{n}=196$ patients) were from elsewhere (Canada and Australia). One trial began in the 1970 s, 28 in the 1980s, 21 in the 1990s, and two thereafter. We directly contacted trialists of 20 out of 29 eligible trials to obtained fidelity data. For the 52 centres, we obtained $95 \%$ complete fidelity data on the team membership and organisation subscales of the fidelity index (mean total fidelity score 6.6, 95\% confidence interval 7.16 to 6.02 ; maximum score 11 , see www.psychiatry.ox.ac.uk/socpsych/bmjmrtable); 42 centres provided data on use of inpatient care before the trial. The 10 centres that did not report it included two services exclusively treating patients with their first psychotic breakdown. ${ }^{\text {w18 w23 }}$

A preliminary meta-analysis showed a small but significant effect in favour of intensive case management but with significant heterogeneity between centres (pooled intervention effect $-0.46,95 \%$ confidence interval -0.84 to $-0.08, \mathrm{P}=0.019$; variation between centres 0.32 , variation between trials 0.32 ).

\section{Meta-regression on days in hospital}

Table 1 shows the meta-regression of mean difference in days per month in hospital (unstandardised) against all covariates except baseline days in hospital (as this covariate was not available for all centres). It shows that the more a case management team is organised like an assertive outreach team, the better it is at reducing time spent in hospital. The regression coefficient for team organisation indicates a decrease

Table 2 | Summary of meta-regression analysis of days in hospital per month ( 42 centres) based on treatment effect estimates: centres with baseline data on hospital use

\begin{tabular}{lcccc}
\multicolumn{1}{c}{$\begin{array}{c}\text { Fidelity } \\
\text { score }\end{array}$} & $\begin{array}{c}\text { Coefficient* } \\
\text { (days/month) }\end{array}$ & SE & $\begin{array}{c}95 \% \mathrm{Cl} \text { for } \\
\text { coefficient }\end{array}$ & P value \\
Organisation & -0.31 & 0.14 & -0.59 to -0.03 & 0.029 \\
\hline $\begin{array}{l}\text { Hospital use } \\
\text { at baseline }\end{array}$ & -0.23 & 0.07 & -0.36 to -0.09 & 0.001 \\
\hline Constant & 1.81 & 0.63 & 0.57 to 3.05 & 0.004 \\
\hline
\end{tabular}

*Variation between centres was 0.53 . No variation between trials. 


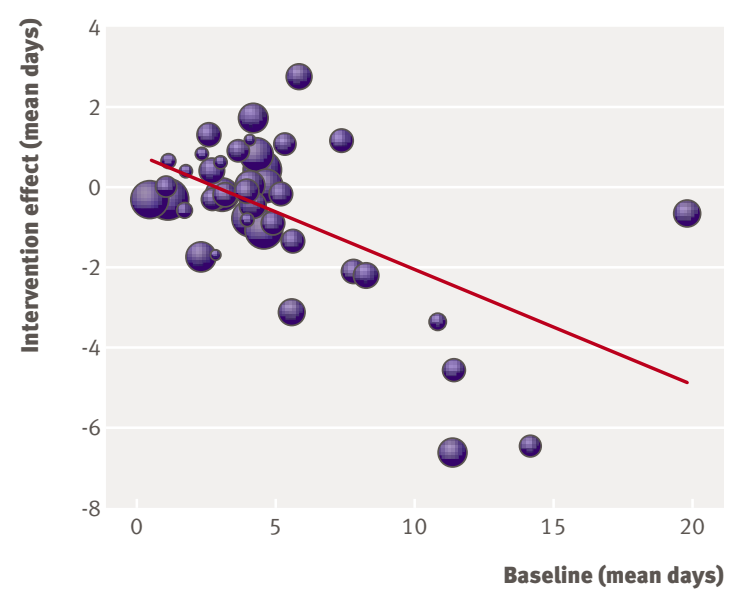

Fig $4 \mid$ Plot of baseline hospital use $v$ mean days per month in hospital. Each circle is proportional to size of centre it represents. Negative treatment effect indicates that intensive case management achieved reduction in mean days in hospital relative to control

of 0.44 (95\% confidence interval 0.72 to 0.17$)$ days in hospital per month for each one point increase on the index organisation scale. The scatter plot of organisation subscore versus mean days in hospital (fig 2) shows that the effect of intensive case management on hospital use is negligible if the team organisation fidelity score is low. No other covariates were significant, including the index team membership subscale (fig 3 ), and there was no evidence of variation within trials between centres. There was, however, continuing evidence of variation between trials, suggesting that differences on the organisation subscore did not entirely explain the heterogeneity between trials.

Table 2 shows the effect of including the covariate of mean days per month in hospital for participants before the trial (available for 42 of the 52 centres). In this model, baseline levels of hospital use and index team organisation subscore were both significant, although the strength of the association between index score and hospital use was reduced compared with that observed in the previous model. There was negligible variation between trials.

In the first sensitivity analysis, we replaced baseline levels of hospital use with mean in the control group as a covariate (see www.psychiatry.ox.ac.uk/socpsych/bmjmrtable). This analysis showed a strong effect of control group mean $(-0.44 ; 95 \%$ confidence interval -0.57 to -0.31 , $\mathrm{P}<0.001$ ), with no other covariates being significant. As this analysis is potentially problematic, we performed a second sensitivity analysis, which modelled means of treatment group rather than the treatment effect with a random intercept and a random coefficient for treatment at the centre and trial level (see www.psychiatry.ox.ac.uk/socpsych/bmjmrtable). When this model was fitted, there was evidence of a strong correlation between the treatment effect and the underlying level of hospital use. The treatment effect was estimated to decrease by 0.31 bed days for each mean bed day difference between centres, in the analysis within studies. The treatment effect decreased by 0.11 bed days for each difference of one bed day between studies. Index organisation score affected the intervention $(-0.47,-0.78$ to $-0.17, \mathrm{P}=0.002)$. No other covariates had significant interactions with intervention group.

When we dropped centres with an imputed SD from the analysis, index organisation score and baseline level of hospital use were no longer significant. This was because a reduction in the number of data points increased the influence of an outlying centre visible in figures 4 and 5 . When we omitted this centre from the analysis the effects remained (see www.psychiatry.ox.ac.uk/socpsych/bmjmrtable).

\section{DISCUSSION}

For severely mentally ill patients, intensive case management works best in trials where participants tend to use a lot of hospital care and less well in trials where they do not. When hospital use is high, intensive case management tends to succeed in reducing it, but it is less successful when hospital use is already low. This is the main reason why the findings of trials on case management are inconsistent. We also found that fidelity to the structure and organisation aspect of the assertive community treatment model explained some of the variation in hospital use between trials. In sensitivity analyses, however, this finding was less robust than explanations based on participants' use of hospital care. Fidelity to the staffing practices of the assertive community treatment model did not, however, explain variation between trials. It is precisely these extra staffing features that have been authorised in the NHS plan ${ }^{4}$ for assertive outreach teams. Some of the control groups in trials of assertive outreach teams with high fidelity might have shared some of their

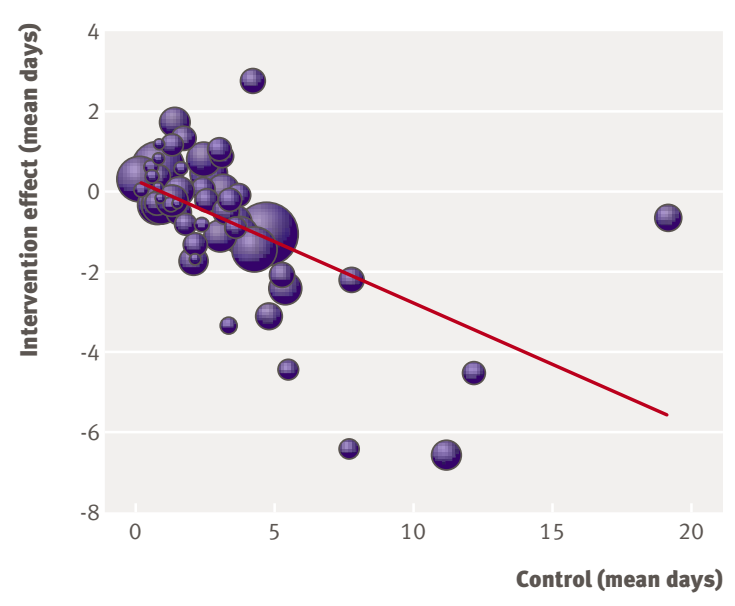

Fig 5 | Plot of control group mean $v$ mean days per month in hospital. Each circle is proportional to size of centre it represents. Negative treatment effect indicates that intensive case management achieved reduction in mean days in hospital relative to control 


\section{WHAT IS ALREADY KNOWN ON THIS TOPIC}

Intensive case management is designed to reduce unnecessary admissions to hospital in severely mentally ill people

Randomised controlled trials of intensive case management have conflicting findings: some have shown reductions in hospital care, while others have found no effect

\section{WHAT THIS STUDY ADDS}

Intensive case management works best in trials where participants tend to use a lot of inpatient care

The effectiveness of intensive case management teams is increased as their organisation reflects the assertive community treatment model, but there is less evidence for the benefits of increased staffing levels intensive case management teams find it difficult to have an impact on hospital use. When community services are poor, it is usually fairly easy for patients to spend long periods of time in hospital, and intensive case management teams find it relatively easy to reduce such wastefulness. There are, however, other explanations. Firstly, low levels of hospital use might indicate that a trial's participants are less severely ill and hence less likely to benefit from intensive case management. Secondly, low levels of hospital use may indicate that it is difficult to obtain admission within the setting in which the trial is being conducted, leaving little scope for the intensive case management team to achieve further reductions.

The main clinical implication of our study is that the introduction of intensive case management teams will not lead to substantial reductions in hospital use in settings where average hospital use is already low. Teams can optimise their ability to reduce hospital use by organising themselves in the manner recommended for assertive community treatment, and by focusing on patients with a history of high hospital use. Replicating staffing requirements of assertive community treatment does not confer measurable benefits. Our study confirms a growing recognition that we should research the practices of teams rather than their labels. ${ }^{22}$ w29

We thank Robert Rosenheck, Michael Neale, Bob Drake, and Gary Bond for providing us with additional data and commenting on the manuscript. We also thank Peter Tyrer, Frank Holloway, Matthew Fiander, Tom Craig, and Merete Nordentoft for additional information concerning the fidelity index.

Contributors: TB is guarantor. TB and MM conceived the review. JC, MD, MM and $\mathrm{AL}$ retrieved the papers and contacted authors. MM and AL prepared the tables and figures. MM, AL, and CR extracted the data, calculated estimates of effects, and dealt with the statistics. All authors read and commented on the paper

Funding: Policy research programme, Department of Health

Competing interests: None declared.

Ethical approval: Not required. have been advanced to explain why intensive management is so inconsistent in reducing hospital care. We have shown that hypotheses based on where and when the trial was conducted can be discounted. We have also shown that fidelity to the assertive community treatment model, insofar as it is important, relates only to aspects of the model concerned with team organisation (in essence, the extent to which case managers work as a team rather than as independent practitioners). Specific staffing features of the assertive community treatment model (such as team size, low caseloads, and professional make up of the team) are not important in reducing hospital use among the team's patients.

\section{Implications of findings}

Why is it that the level of hospital use among a trial's participants is so important in determining the effectiveness of intensive case management? We think that low levels of hospital use are a proxy for good community services. Where community services are good, hospital care is used sparingly and only when absolutely necessary. Under such circumstances even
1 NASHMPD Research Institute. Implementation of evidence-based services by state mental health authorities. Community Support Network News 1997;11:3-5.

2 Bond GR, Drake RE, Mueser KT, Latimer E. Assertive community treatment for people with severe mental illness: critical ingredients and impact on patients. Dis Manag Health Outcomes 2001;9:141-59.

3 Burns T, Fioritti A, Holloway F, Malm U, Rossler W. Case managemen and assertive community treatment in Europe. Psychiatr Serv 2001;52:631-36.

4 Department of Health. A national service framework for mental health. London: Department of Health, 1999.

5 Holloway F. Case management for the mentally ill: looking at the evidence. Int J Soc Psychiatry 1991;37:2-13.

6 Tyrer P. Whither community care? Br J Psychiatry 1998;173:359-60.

7 Marshall M, Creed F. Assertive community treatment-is it the future of community care in the UK? Soc Psychiatry Psychiatr Epidemiol 2000;12:191-96.

8 McGovern D, Owen A. Intensive case management for severe psychotic illness. Lancet 1999;354:1384.

9 Tyrer P. Are small caseloads beautiful in severe mental illness? $\mathrm{Br}$ Psychiatry 2000;177:386-7.

10 Tyrer P. Effectiveness of intensive treatment in severe mental illness. BrJ Psychiatry 2000;176:492-3.

11 Burns T, Catty J, Watt H, Wright C, Knapp M, Henderson J. International differences in home treatment for mental health 2002;181:375-82. problems: results of a systematic review. Br J Psychiatry 
12 Marshall M, Gray A, Lockwood A, Green R. Case management for people with severe mental disorders. Cochrane Database Syst Rev 1998;(2):CD000050.

13 Marshall M, Lockwood A. Assertive community treatment for people with severe mental disorders. Cochrane Database Syst Rev 1998;(2) CD001089.

14 Higgins JPT, Green S, eds. Selection bias. Cochrane handbook for systematic reviews of interventions 4.2.5. Chichester: Wiley, 2005.

$15 \mathrm{McGrew} \mathrm{JH}$, Bond GR, Dietzen LL, Salyers M. Measuring the fidelity of implementation of a mental health program model. J Consult Clin Psychol 1994;62:670-8.

16 Burns T, Knapp M, Catty J, Healey A, Henderson J, Watt H, et al. Home treatment for mental health problems: a systematic review. Health Technol Assess 2001;5:1-139.

17 Catty J, Burns T, Knapp K, Watt H, Wright C, Henderson J, et al. Home treatment for mental health problems: a systematic review. Psychol Med 2002;32:383-401.
18 Burns T, Fiander M, Kent A, Ukoumunne OC, Byford S, Fahy T, et al. Effects of caseload size on the process of care of patients with severe psychotic illness: report from the UK700 trial. Br J Psychiatry 2000;177:427-33.

19 Rabe-Hesketh S, Pickles A, Skrondal S. GLLAMM manual. Technical report 2001/01. London: Department of Biostatistics and Computing, Institute of Psychiatry, King's College, 2001.

20 Burns T, Creed F, Fahy T, Thompson S, Tyrer P, White I. Intensive versus standard case management for severe psychotic illness: a randomised trial. Lancet 1999;353:2185-9.

21 Killaspy H, Bebbington P, Blizard R, Johnson S, Nolan F, Pilling S, et al. The REACT study: randomised evaluation of assertive community treatment in north London. BMJ 2006;332:815-20.

22 Wright C, Catty J, Watt H, Burns T. A systematic review of home treatment services: classification and sustainability. Soc Psychiatry Psychiatr Epidemiol 2004;39:7, 89-96.

Accepted: 5 June 2007 